\title{
Control Exitoso del dolor oncológico crónico por cáncer de pulmón con el bloqueo del plano del erector espinal (BPEE) en un servicio de neumología. Reporte de caso
}

\author{
Successful control of chronic cancer pain from lung cancer with erector spinae \\ plane (ESP) block in a pulmonology department. Case report
}

\author{
Samuel Pecho-Silva ${ }^{1,2}$, Ana Claudia Navarro-Solsol ${ }^{3}$, David L. Tello-Rojas ${ }^{4}$, Vicky Panduro-Correa ${ }^{4}{ }^{*}$ \\ Hospital Nacional Edgardo Rebagliati Martins. Lima, Perú. \\ Maestría en Epidemiología Clínica y Bioestadística, Universidad Científica del Sur. Lima, Perú. \\ Universidad Nacional de Ucayali. Pucallpa, Perú. \\ $4 \quad$ Facultad de Medicina, Universidad Nacional Hermilio Valdizán. Huánuco, Perú.
}

Fuentes de financiamiento: El estudio fue de carácter autofinanciado.

Conflictos de interes: Ninguno declarado por los autores.

Fecha de ingreso: 20 de septiembre de 2020 / Fecha de aceptación: 12 de enero de 2021

\begin{abstract}
Cancer pain is defined as an unpleasant sensory and emotional experience. $90 \%$ of patients with terminal cancer present pain. An alternative for the management of pain located in the thorax is erector spinae plane (ESP) block guided by real-time ultrasound. However, its use in the management of oncological chest pain is not very common, with few reports in this regard, with most of these reports always performed by anesthesiologists. We describe our first experience performing a real-time guided ESP block using ultrasound performed by a trained pulmonologist in a 64 years-old male with lung adenocarcinoma stage IVa with sternum metastases and multiple left costal arches associated with left pleural effusion due to pleural metastases. We found ESP block to be safe for the patient as well as effective in reducing pain measured by Visual Analogic Score and was able to reduce the use of systemic analgesics.
\end{abstract}

Key words: Cancer pain, erector spinae plane block, real-time ultrasound.

\section{RESUMEN}

El dolor es una experiencia sensorial y emocional desagradable. El $90 \%$ de los pacientes con cáncer terminal presentan dolor y una alternativa para el tratamiento del dolor localizado en el tórax es el bloqueo del plano del erector espinal de la columna (BPEE) guiado por ultrasonido en tiempo real. Sin embargo, su uso en el tratamiento del dolor oncológico no es común y hay pocos informes al respecto, la mayoría realizados por anestesiólogos. Describimos nuestra primera experiencia realizando un BPEE guiado en tiempo real con ultrasonido y realizado por un neumólogo en un varón de 64 años con adenocarcinoma de pulmón en estadio IVa, con metástasis en el esternón y múltiples arcos costales izquierdos y derrame pleural izquierdo debido a metástasis pleural. Descubrimos que el bloqueo BPEE fue seguro y efectivo para reducir el dolor medido por la puntuación de Escala Visual Analógica y fue capaz de reducir el uso de analgésicos sistémicos.

Palabras clave: Dolor oncológico, bloqueo del plano del erector espinal de la columna, ultrasonido en tiempo real.

vpanduro@unheval.edu.pe

https://orcid.org/0000-0002-2445-4854 


\section{Introducción}

$E^{\prime}$ dolor oncológico es definido como una experiencia sensorial y emocional desagradable. Cerca del 90\% de pacientes con cáncer terminal lo van a padecer y el tratamiento sigue siendo iniciar con antiinflamatorios no esteroideos hasta llegar a opioides y opiáceos menores y mayores enterales y/o parenterales. Aunque se puede lograr el control del dolor las reacciones adversas son frecuentes[1]. Una alternativa reciente para el manejo del dolor localizado en el tórax es el BPEE guiado con ecografía en tiempo real; técnica desarrollada y descrita inicialmente por Forero[2] y que recientemente ha sido materia de revisión[3],[4]. Su uso se ha extendido ampliamente al manejo del dolor nociceptivo, neuropático, posherpético y postoperatorio entre otros[5]-[8].

El BPEE está siendo estudiada por diversas especialidades médicas por su prometedor desempeño en el manejo del dolor en varias regiones del cuerpo[9]. La impregnación a varios niveles sensibles de generar analgesia[10] la ha posicionado como una opción fácil y accesible para que, incluso médicos no anestesiólogos puedan brindar analgesia a pacientes en escenarios aún fuera de sala de operaciones[11]. Su uso para el manejo del dolor oncológico del tórax no es muy habitual, son pocas las revisiones al respecto, generalmente reportes de series de casos[12]. En los hospitales del Perú y en general de Latinoamérica son muy pocos los pacientes con dolor oncológico que acceden al BPEE por la especialidad de anestesiología, y esta técnica al parecer no ha sido realizada dentro de los servicios de neumología que cuentan con ecógrafo. Este caso clínico describe un BPEE guiado en tiempo real con ecografía dentro de un servicio de neumología.

\section{Caso clínico}

Paciente varón de 64 años de edad con diagnóstico de adenocarcinoma de pulmón Estadío Clínico IVa con metástasis en esternón y múltiples arcos costales izquierdos asociados a derra- me pleural izquierdo por metástasis pleural con un EVA (escala visual analógica) de 10/10 recibiendo opioides mayores sin lograr alcanzar el control total del dolor. Luego de obtener el consentimiento informado del paciente, se procedió a utilizar un ecógrafo inalámbrico portátil marca Sonostar (transductor: electronic array, modelo Q1) y usando un transductor lineal multifrecuencia (7,5 - $10 \mathrm{Mhz}$ ), se realizó una evaluación ecográfica con el paciente en decúbito ventral con la cabeza hacia el operador para identificar la vértebra T5 y su proceso transverso izquierdo (transductor en posición transversal al paciente inicialmente). Luego se rotó el transductor $90^{\circ}$ a una posición longitudinal con respecto al paciente (Figura 1) y se desplazó lateralmente una distancia aproximada de $3 \mathrm{~cm}$ pudiéndose identificar a los músculos: trapecio, romboide mayor y erector espinal (desde la superficie hacia la profundidad de la pared costal), así como el proceso transverso y la línea pleural. Se procedió a realizar la asepsia de la zona y con una jeringa con $24 \mathrm{~mL}$ de una solución que contenía anestésico local (20 mL de xilocaína al 1\% sin epinefrina y 4 $\mathrm{mL}$ con $8 \mathrm{mg}$ de dexametasona) y una aguja de 0,8 x $25 \mathrm{~mm}$ y $21 \mathrm{G}$ se ingresó mediante la técnica "en plano" en dirección cefalocaudal hasta llegar por debajo del músculo erector espinal y por encima del proceso transverso. Cuando la aguja se encontró posicionada bajo guía ecográfica en tiempo real se inyectaron 2 $\mathrm{mL}$ de la solución y al evidenciar que se lograba una correcta infiltración interfascial se inyectaron los $22 \mathrm{~mL}$ restantes, todo bajo la guía ecográfica en tiempo real.

No se presentaron alteraciones en las funciones vitales durante el procedimiento ni luego de éste. El paciente no manifestó ninguna molestia durante el procedimiento. El EVA a los 20 minutos fue de 6 puntos y a los 45 minutos fue de 4 puntos. A las 2 h el EVA fue de 0 puntos para todo el dolor del tórax (arcos costales, esternón, cavidad pleural). Durante las siguientes $48 \mathrm{~h}$ el paciente no necesitó del uso de analgésicos enterales o parenterales de ningún tipo para el control del dolor. Luego de ese periodo el paciente reinició opiáceos. No repetimos el procedimiento nuevamente en el paciente. Finalmente 3 semanas después el paciente falleció debido al estado terminal del cáncer de pulmón.

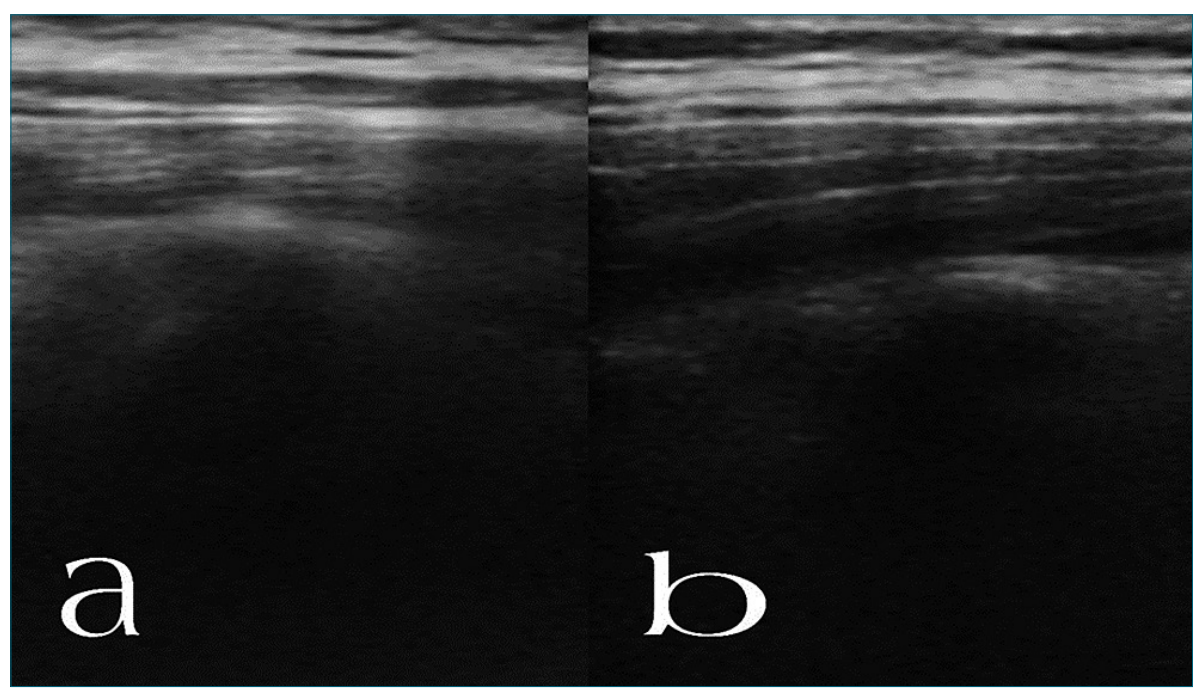

Figura 1. (a) Identificación de los siguientes músculos: trapecio, romboide mayor y erector espinal (desde la superficie hasta la profundidad de la pared costal), así como el proceso transverso y la línea pleural; (b) Solución inyectada debajo del músculo erector espinal y por encima del proceso transversal. 


\section{Discusión}

Este procedimiento fue realizado fuera de centro quirúrgico, por especialistas neumólogos con entrenamiento en ultrasonido, sin mayor alteración del estado general del paciente. El BPEE posee una mayor penetración a tejidos nerviosos como el espacio peridural, lo que conlleva un riesgo de complicacionesAunque el BPEE ha sido encontrado más seguro que el bloqueo paravertebral o el epidural torácico, se necesitan grandes ensayos clínicos donde se evidencie la seguridad y la frecuencia de complicaciones de este procedimiento[9]-[11]. El procedimiento debería realizarse con una monitorización básica, para facilitar la administración consuetudinaria de este bloqueo en pacientes oncológicos, todo esto en un proceso de estandarización de su uso; se debería realizar una lista de verificación para mejorar la seguridad del procedimiento[13].

Los volúmenes de anestésico local usados varían de los 20 a $30 \mathrm{~mL}$ sea en su forma pura o diluida[10],[14], por lo que se deben investigar siempre signos de toxicidad por anestésicos locales y tener un plan de trabajo en caso se presente. La realización del bloqueo en un paciente ambulatorio haría necesario el desarrollo de un ambiente de procedimientos para que se realice de forma segura. Se ha reportado neumotórax producido durante el bloqueo, aunque esta complicación es considerada rara[10], dada la distancia que tiene el punto de infiltración hasta la pleura parietal y porque en todo momento se visualiza la aguja y se controla su desplazamiento.

El volumen anestésico usado en este paciente se encuentra comprendido entre lo descrito por otros autores, la lidocaína no es el anestésico local de elección para el dolor crónico, el uso de catéter habría ayudado a dar un mantenimiento en el manejo del dolor de este paciente[12],[14].

Todavía se encuentra en investigación todas las aplicaciones a la que pudiera dar lugar el uso de este bloqueo, pero ya se han descrito, principalmente a través de reporte de casos, su uso para manejo del dolor crónico oncológico por cáncer de mamas y pulmones[12], dolor posoperatorio en tórax y otras patologías y procedimientos vinculados al tórax. Además, nefrolitotomías, cirugía vertebral lumbar, cirugía laparoscópica abdominal alta, entre otras[10],[15]. Este paciente presentaba metástasis torácicas, pero ya se han reportado casos exitosos de manejo del dolor en zonas extratorácicas.

Este bloqueo puede brindar analgesia no sólo de la pared torácica o abdominal, sino además a la víscera, por lo que tendría una mejor calidad analgésica y la colocaría con una aparente ventaja por delante de bloqueos como el bloqueo paravertebral o el retrolaminar y con menor complejidad técnica que el bloqueo peridural[9],[11],[15]-[17]; cómo en el presente caso logrando resolver de manera exitosa el dolor oncológico severo por 48 horas, pasando la evaluación del dolor de 10 a 0 en pocos minutos y manteniéndose así por ese período. Esta aparente facilidad se debe, en parte, a la extensión o difusión analgésica en los dermatomas desde la zona infiltrada, esto abarca 3 a 4 dermatomas tanto en sentido cefálico como caudal, extendiéndose además lateralmente[10],[15]. El bloqueo puede realizarse con un bolo simple (single shot) o de forma continua con la colocación de un catéter peridural en la zona de infiltración[15].

Los bloqueos en general han reducido el uso de analgésicos en el paciente con cáncer, incluyendo los opioides. De esta forma han mejorado el estado de alerta de los pacientes, la desaturación entre otros efectos adversos asociados; además de todo esto maneja el dolor refractario a los fármacos de uso habitual en cáncer. En el caso del BPEE aún faltan estudios vinculados al dolor crónico de tipo oncológico, a pesar de esta falta de evidencia los reportes de casos muestran un descenso considerable de la intensidad del dolor con respecto a los niveles previos al bloqueo[15], un detalle interesante es que el dolor oncológico se muestra aliviado de manera sostenida aún meses después de un procedimiento con un bloqueo simple (single shot), es decir sin el uso de catéter[9]. Nuestro paciente en cuestión recibió un bloqueo "single shot" sin catéter con una dilución al 1\% de lidocaína, sin embargo, el dolor retornó a las 48 h. La selección de otro anestésico local como la bupivacaína habría mejorado este resultado aún más si se hubiera combinado con dexmedetomidina.

Finalmente, el manejo del dolor resulta en una obligación moral para el médico y no debe abandonarse ningún paciente sin manejo adecuado, por lo que el BPEE debería considerarse como alternativa de manejo en paciente con dolor oncológico de difícil tratamiento y lograr su implementación en unidades que manejan este tipo de pacientes.

\section{Referencias}

1. Khosravi Shahi P, del Castillo Rueda A, Pérez Manga G. Manejo del dolor oncológico. Med Interna. 2007 Nov;24(11):554-7.

2. Forero M, Adhikary SD, Lopez H, Tsui C, Chin KJ. The Erector Spinae Plane Block: A Novel Analgesic Technique in Thoracic Neuropathic Pain. Reg Anesth Pain Med. 2016 Sep-Oct;41(5):621-7. https://doi.org/10.1097/AAP.0000000000000451 PMID:27501016

3. Kot P, Rodriguez P, Granell M, Cano B, Rovira L, Morales J, et al. The erector spinae plane block: a narrative review. Korean J Anesthesiol. 2019 Jun;72(3):209-20. https://doi.org/10.4097/ kja.d.19.00012 PMID:30886130

4. Tulgar S, Ahiskalioglu A, De Cassai A, Gurkan Y. Efficacy of bilateral erector spinae plane block in the management of pain: current insights. J Pain Res. 2019 Aug;12:2597-613. https://doi. org/10.2147/JPR.S182128 PMID:31695476

5. Marciniak D, Kelava M, Hargrave J. Fascial plane blocks in thoracic surgery: a new era or plain painful? Curr Opin Anaesthesiol. 2020 Feb;33(1):1-9. https://doi.org/10.1097/ ACO.0000000000000803 PMID:31688087

6. Hu B, Zhou H, Zou X. The erector spinae plane block (ESPB) for non-intubated video-assisted thoracoscopic surgery. J Clin Anesth. 2019 May;54:50-1. https://doi.org/10.1016/j.jclinane.2018.10.032 PMID:30391456

7. Gürkan Y, Aksu C, Kuş A, Yörükoğlu UH. Erector spinae plane block and thoracic paravertebral block for breast surgery compared to IV-morphine: A randomized controlled trial. J Clin Anesth. 2020 Feb;59:84-8. https://doi.org/10.1016/j.jclinane.2019.06.036 PMID:31280100

8. Bang S, Chung K, Chung J, Yoo S, Baek S, Lee SM. The erector spinae plane block for effective analgesia after lung lobectomy: three cases report. Medicine (Baltimore). 2019 Jul;98(29):e16262. https://doi.org/10.1097/MD.0000000000016262 PMID:31335674

9. Urits I, Charipova K, Gress K, Laughlin P, Orhurhu V, Kaye AD, et 
al. Expanding Role of the Erector Spinae Plane Block for Postoperative and Chronic Pain Management. Curr Pain Headache Rep. 2019 Aug;23(10):71. https://doi.org/10.1007/s11916-019-0812-y PMID:31372769

10. Onishi E, Toda N, Kameyama Y, Yamauchi M. Comparison of Clinical Efficacy and Anatomical Investigation between Retrolaminar Block and Erector Spinae Plane Block. BioMed Res Int. 2019 Mar;2019:2578396. https://doi.org/10.1155/2019/2578396 PMID:31032339

11. El-Boghdadly K, Pawa A. The erector spinae plane block: plane and simple. Anaesthesia. 2017 Apr;72(4):434-8. https://doi. org/10.1111/anae.13830 PMID:28188611

12. Kalagara HK, Deichmann P, Brooks B, Nagi P, Kukreja P. T1 Erector Spinae Plane Block Catheter As a Novel Treatment Modality for Pancoast Tumor Pain. Cureus. 2019 Nov;11(11):e6092. https://doi.org/10.7759/cureus.6092 PMID:31857924

13. Henshaw DS, Turner JD, Dobson SW, Douglas Jaffe J, Wells Reynolds J, Edwards CJ, et al. Preprocedural checklist for re- gional anesthesia: impact on the incidence of wrong site nerve blockade (an 8-year perspective). Reg Anesth Pain Med. 2019 Jan;44(2):rapm-2018-000033. https://doi.org/10.1136/rapm2018-000033 PMID:30636720

14. De Cassai A, Tonetti T. Local anesthetic spread during erector spinae plane block. J Clin Anesth. 2018 Aug;48:60-1. https://doi. org/10.1016/j.jclinane.2018.05.003 PMID:29753992

15. Krishnan S, Cascella M. Erector Spinae Plane Block. StatPearls [Internet]Treasure Island (FL): StatPearls Publishing; 2020.[ [cited 2020 Aug 12]], Available from http://www.ncbi.nlm.nih.gov/ books/NBK545305/

16. Hamilton DL, Manickam B. Erector spinae plane block for pain relief in rib fractures. Br J Anaesth. 2017 01;118(3):474-5. https:// doi.org/10.1093/bja/aex013.

17. Schwartzmann A, Peng P, Maciel MA, Forero M. Mechanism of the erector spinae plane block: insights from a magnetic resonance imaging study. Can J Anaesth. 2018 Oct;65(10):1165-6. https://doi.org/10.1007/s12630-018-1187-y PMID:30076575 